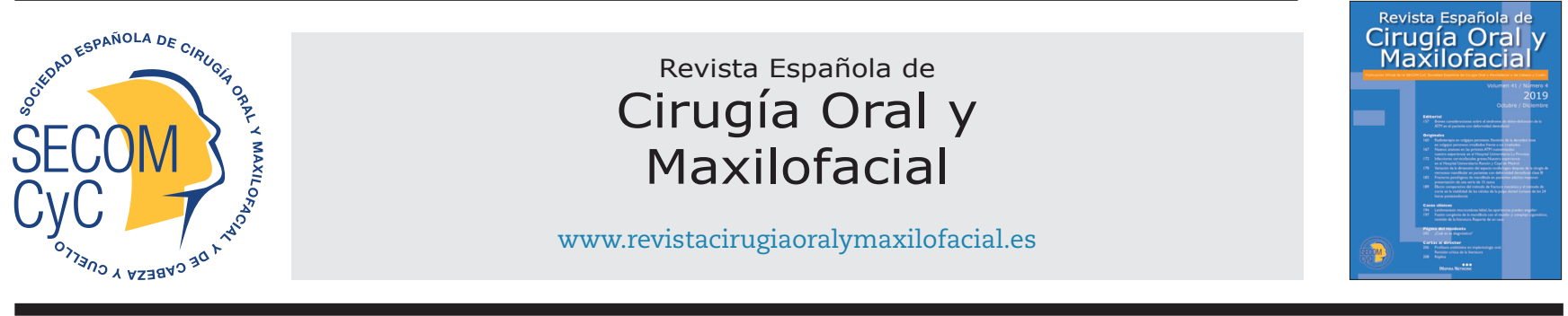

\title{
Original
}

\section{Fracturas patológicas de mandíbula en pacientes adultos mayores: presentación de una serie de 15 casos}

\section{Marcelo Oscar Miniguttia ${ }^{*}$, Emilio Andrés Ramos ${ }^{a}$, Diego Caruso ${ }^{b}$, Juan Manuel Muiño Ludmila Betsabé Antonellia y Maximiliano Diamante ${ }^{a}$}

aServicio de Cirugía Maxiolofacial, Hospital Dr. César Milstein, Buenos Aires, Argentina. ${ }^{b}$ Departamento de Investigación Clínica, Hospital Dr. César Milstein, Buenos Aires, Argentina.

INFORMACIÓN DEL ARTÍCULO

Historia del artículo:

Recibido el 18 de marzo de 2018

Aceptado el 17 de enero de 2019

Palabras clave:

Fractura patológica, mandíbula, adulto mayor, osteonecrosis asociada a medicamentos.

\section{R E S U M E N}

Introducción: Las fracturas patológicas de mandíbula representan menos del $2 \%$ del total de las fracturas de mandíbula y frecuentemente afectan a pacientes adultos mayores. En pacientes mayores de 65 años estas fracturas podrían presentar características diferentes a las de la población general por su asociación con múltiples comorbilidades.

Materiales y métodos: Presentamos un estudio descriptivo retrospectivo de una serie de 15 casos de fracturas patológicas de mandíbula. Solo se incluyeron pacientes mayores de 65 años y se recolectaron las siguientes variables: sexo, edad, etiología, localización, tratamiento y complicaciones postoperatorias. Además se llevó a cabo una revisión de la literatura en PubMed acerca de fracturas patológicas de mandíbula.

Resultados: La edad promedio fue de 72 años (rango: 65-85) y el $67 \%$ ( $n=10)$ de los casos fueron pacientes de sexo femenino. La etiología más frecuente fue la osteonecrosis asociada a medicamentos (ONAM) (40\%). La tasa de complicaciones postoperatorias fue del $53 \%(8 / 15)$. En la revisión de la bibliografía se identificaron 24 artículos acerca de fracturas patológicas de mandíbula. Solo tres publicaciones incluyen más de 10 pacientes. Ninguna incluye pacientes adultos mayores exclusivamente.

Conclusiones: En nuestra serie encontramos que la etiología más frecuente fue la ONAM. Estos pacientes pueden ser tratados de forma exitosa siguiendo los algoritmos propuestos para la población general, aunque la tasa de complicaciones es elevada (53\%).

\footnotetext{
*Autor para correspondencia:

Correo electrónico: mom2687@gmail.com (Marcelo Oscar Minigutti).

DOI: 10.20986/recom.2019.1041/2019
}

1130-0558/@ 2019 SECOM. Publicado por Inspira Network. Este es un artículo Open Access bajo la licencia CC BY-NC-ND (http:// creativecommons.org/licenses/by-nc-nd/4.0/). 


\section{Pathological fractures of the mandible In elderly patients: a series of 15 cases}

\section{A B S T R A C T}

Keywords:

Pathologic fracture, mandible, elderly, medication-related osteonecrosis of the jaw.
Introduction: Pathological fractures of the mandible account for fewer than $2 \%$ of all mandibular fractures and often affect elderly patients. In patients over 65 years of age, these fractures may present features that are different from those observed in the general population because of their association with multiple comorbidities.

Materials and methods: In this paper, we present a retrospective study of a series of 15 cases of pathological fractures of the mandible. Only patients over 65 years old were included, and the following variables were collected: sex, age, etiology, localization, treatment and postoperative complications. Additionally, we conducted a literature review in PubMed on pathological fractures of the mandible.

Results: The mean age was 72 years (range 65-85) and $67 \%(n=10)$ were female patients. The most common etiology was medicine-related osteonecrosis of the jaws (40\%). The postoperative complication rate was $53 \%(8 / 15)$. We identified 24 articles assessing pathological fractures of the mandible in our review of the literature. Only three articles included more than 10 patients. None of them related exclusively to elderly patients.

Conclusions: In our series, the most frequently encountered etiology was medicine-related osteonecrosis of the jaws. These patients can be treated successfully following the same algorithms used in the general population, albeit the complication rate is high (53\%).

\section{INTRODUCCIÓN}

Las fracturas patológicas de mandíbula se definen como aquellas que ocurren a través de una lesión o porción debilitada de la estructura ósea ${ }^{1}$. Representan menos del $2 \%$ del total de las fracturas de la mandíbula ${ }^{2}$ y frecuentemente afectan a pacientes adultos mayores. Considerando el crecimiento poblacional actual, se estima que el $22 \%$ de la población mundial será mayor de 65 años en $2030^{3}$. Esta tendencia se mantiene en los países de altos ingresos y, para ese mismo año, la población de adultos mayores alcanzará aproximadamente el $25 \%{ }^{3}$.

En pacientes mayores de 65 años las fracturas patológicas de la mandíbula podrían presentar características diferentes a las de la población general por su asociación con múltiples comorbilidades, tales como el inmunocompromiso, la desnutrición, el enolismo, el tabaquismo ${ }^{4}$, la diabetes, la hipertensión arterial y las patologías cardiacas 5 . Por otro lado, el incremento de edad y la presencia de comorbilidades asociadas aumentan la morbimortalidad postquirúrgica ${ }^{6}$. Las series de casos publicadas en la bibliografía acerca de fracturas patológicas de mandíbula son escasas y ninguna de estas incluye exclusivamente pacientes adultos mayores. El objetivo de este trabajo es evaluar las características de estas fracturas en una serie de pacientes mayores de 65 años. Se presenta además una revisión narrativa de la bibliografía.

\section{MATERIALES Y MÉTODOS}

Presentamos un estudio descriptivo retrospectivo de una serie de 15 casos de fracturas patológicas de mandíbula. La identificación de casos se llevó a cabo a partir de la base de datos del Servicio de Cirugía Maxilofacial del Hospital Dr. Cé- sar Milstein. Después de su identificación, se revisaron las historias clínicas en papel de los casos ingresados entre julio de 2010 y julio de 2017. Se incluyeron pacientes mayores de 65 años en los que el trazo de la fractura se encontraba asociado a una lesión o a una porción debilitada de la mandíbula. Se excluyeron aquellas ocurridas en mandíbulas atróficas. Se recolectaron las siguientes variables: sexo, edad, etiología, localización, tratamiento y complicaciones postoperatorias. Además se recolectó información acerca del tratamiento de las complicaciones, de la evolución y del tiempo de seguimiento. La elección del tratamiento se llevó a cabo siguiendo el algoritmo propuesto por Coletti y Ord (Figura 1) ${ }^{7}$. En pacientes sin alteraciones sistémicas, con tejido óseo normal y con ade-

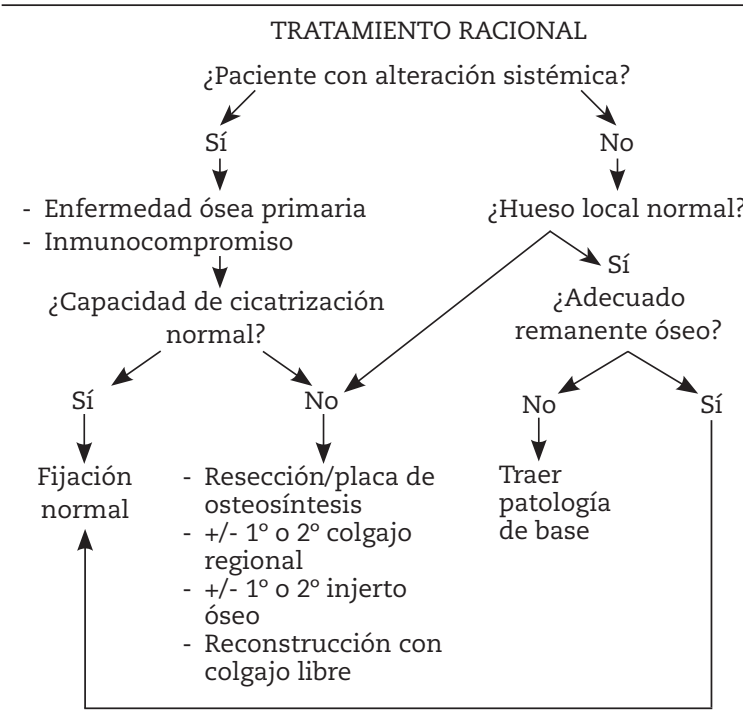

Figura 1. Algoritmo de tratamiento de fracturas patológicas de mandíbula (modificado de Coletti y Ord, 200877). 
cuado remanente óseo, al igual que en pacientes con inmunocompromiso o patología ósea primaria pero sin alteración de la cicatrización ósea, se realizó fijación habitual con osteosíntesis rígida. En pacientes con enfermedad ósea primaria o inmunocompromiso, y que además presentaban alteración de la capacidad de la cicatrización ósea, se llevó a cabo la resección y colocación de placas de osteosíntesis en combinación o no con colgajos regionales o libres. Se siguieron las mismas pautas de tratamiento para pacientes sin alteraciones sistémicas pero con remanente óseo inadecuado.

Adicionalmente se llevó a cabo una revisión narrativa de la bibliografía en PubMed empleando la siguiente estrategia: "mandibular fractures"[MeSH Terms] AND "pathological"[All Fields] AND ("humans"[MeSH Terms] AND English[lang]). Se incluyeron artículos en inglés, sobre humanos, publicados entre enero de 1965 y agosto de 2017.

\section{RESULTADOS}

Se identificaron 15 casos de fracturas patológicas de mandíbula en pacientes adultos mayores. Los datos referidos a sexo, edad, etiología, tratamiento y complicaciones, tratamiento secundario, evolución y tiempo de seguimiento se detallan en la Tabla I. La edad promedio fue de 72 años (rango: 65-85) y el $67 \%$ $(n=10)$ de los casos fueron pacientes de sexo femenino. La etiología más frecuente fue la osteonecrosis asociada a medicamentos (ONAM) (40\%), seguida por el carcinoma de células escamosas (CCE) (27\%) y la osteorradionecrosis (ORN) $(20 \%)$. Un caso (6\%) estuvo asociado a mieloma múltiple y otro (6\%) a un quiste odontogénico inflamatorio. El sitio anatómico más frecuentemente afectado fue el cuerpo mandibular (73\%), seguido por la región parasinfisiaria (20 \%) y finalmente el ángulo mandibular (7\%). Con respecto al tratamiento, en 9 pacientes (60\%) se llevó a cabo la reducción abierta con fijación rígida, en 3 pacientes $(20 \%)$ se realizó la resección mandibular con colocación de osteosíntesis rígida y colgajos regionales, en un paciente (6\%) se realizó la resección mandibular con colocación de osteosíntesis rígida y en otro (6 \%) se utilizó un colgajo libre. Finalmente, un paciente fue tratado con antibióticos sistémicos y antisépticos locales debido a la imposibilidad de ser intervenido bajo anestesia general. La tasa de complicaciones postoperatorias fue del $53 \%$ (8/15). De estas, el $50 \%$ (4/8) presentó exposición de placa de osteosíntesis, el 25 \% (2/8) necrosis del colgajo, el $13 \%$ (1/8) fracaso del material de osteosíntesis y el $13 \%$ (1/8) fístula orocutánea. El tratamiento secundario realizado sobre los pacientes que presentaron complicaciones y su evolución se especifica en la Tabla I. Se identificaron 24 artículos acerca de fracturas patológicas de mandíbula. Solo tres publicaciones incluyen más de 10 pacientes $^{4,7,8}$. Ninguna incluye pacientes adultos mayores exclusivamente. En la Tabla II se resumen los principales hallazgos de la revisión de la bibliografía.

\section{DISCUSIÓN}

Según los reportes de la bibliografía las etiologías más frecuentes de fracturas patológicas de mandíbula son: ORN $(33,3 \%)$, osteomielitis (20,5 \%), ONAM (11,1\%), CCE $(7,6 \%)$, quistes $(6,8 \%)$, tumores benignos $(5,1 \%)$ y metástasis $(4,2 \%)$.
En nuestra serie, la ONAM (40\%) fue la etiología más frecuente, seguida por el CCE $(26,6 \%)$ y la ORN (20\%). Estas diferencias podrían deberse a la mayor incidencia de ONAM en la población de adultos mayores en comparación con la población general y a la disminución de la incidencia de ORN por el uso creciente de la radioterapia de intensidad modulada. Cabe señalar, además, que nuestro centro recibe derivación de patología maligna de diversas localidades del resto del país. Esta situación, sumada a la agresividad de la lesión, podría explicar el hecho de que el CCE sea la segunda etiología más frecuente de nuestra serie.

El manejo de las fracturas mandibulares patológicas constituye un desafío para el cirujano maxilofacial. Además de la reducción y la fijación de los trazos de fractura, es necesario el tratamiento de la lesión asociada. Coletti y $\operatorname{Ord}^{7}$ propusieron un algoritmo en el que la decisión terapéutica se toma en función de la condición del tejido óseo, el estado sistémico del paciente y la capacidad de cicatrización de los tejidos. Estos autores informaron de una tasa de complicaciones del $40 \%$ sobre 44 casos de fracturas mandibulares patológicas. Las complicaciones informadas en esta serie fueron: muerte por la enfermedad (6 casos), exposición de placas de osteosíntesis (5 casos), fracaso de la osteosíntesis (4 casos), infección de la osteosíntesis (3 casos), maloclusión (1 caso), infección de injerto (1 caso) y pérdida de seguimiento (1 caso). El resto de las publicaciones disponibles en la bibliografía no brindan información detallada sobre este punto. En nuestra serie la tasa de complicaciones fue del $53 \%$ empleando el algoritmo de tratamiento propuesto por Coletti y $\operatorname{Ord}^{7}$. Estos porcentajes duplican la tasa de complicaciones reportadas en fracturas mandibulares no patológicas ${ }^{28,29}$. La presencia de comorbilidades múltiples en este grupo de pacientes y la complejidad de las patologías subyacentes (estadios avanzados de ONAM, ORN y CCE) podrían explicar este fenómeno. Con respecto a este último punto, cabe mencionar que el $73 \%$ ( $n=11$ ) de los casos incluidos en nuestro estudio fueron pacientes oncológicos.

En el subgrupo de pacientes con ONAM se produjo la exposición de material de osteosíntesis en el 50 \% de los casos (3 pacientes). El tratamiento secundario consistió en la aplicación de cuidados locales de la herida mediante lavados con solución antiséptica en combinación o no con antibióticos por vía oral. Dos casos evolucionaron favorablemente y uno continuó con tejido óseo necrótico expuesto. En los pacientes con diagnóstico de ONAM, el 83 \% (5 casos) evolucionó hacia la cicatrización completa en un periodo de seguimiento de 14 meses. Coletti y Ord informaron de las mismas de tasas de complicaciones en este grupo de pacientes ${ }^{7}$. En coincidencia con lo informado por otros autores ${ }^{4,8}$, la peor evolución se produjo en el subgrupo de pacientes con diagnóstico de CCE. Todos ellos presentaban estadios avanzados de la enfermedad y fallecieron tras un periodo de seguimiento promedio de 8 meses. En concordancia con los resultados referidos en la bibliografía ${ }^{7,8}$, la tasa de complicaciones en pacientes con ORN fue elevada (66\%). A pesar de ello, dos de tres pacientes (66\%) evolucionaron favorablemente después del tratamiento secundario en un periodo de 11 meses de seguimiento.

A diferencia de lo que ocurre con las fracturas no patológicas, cuya localización más frecuente es el cuello de cóndilo y el ángulo ${ }^{30}$, en nuestra serie existió un claro predominio de las fracturas de cuerpo mandibular (73\%). 
Tabla I. Sexo, edad, etiología, localización, tratamiento y complicaciones del tratamiento

\begin{tabular}{|c|c|c|c|c|c|c|c|c|}
\hline $\mathrm{N}$ & $\begin{array}{l}\text { Sexo, } \\
\text { Edad }\end{array}$ & Etiología & Localización & Tratamiento & Complicaciones & $\begin{array}{l}\text { Tratamiento } \\
\text { Secundario }\end{array}$ & Evolución & Seguimiento \\
\hline 1 & F, 66 & $\begin{array}{c}\text { CCE } \\
\text { (estadio IVa) }\end{array}$ & Cuerpo & $\begin{array}{c}\text { Resección + RAFR } \\
\text { + CMPM }\end{array}$ & - & - & $\begin{array}{l}\text { Muerte por } \\
\text { enfermedad }\end{array}$ & 8 meses \\
\hline 2 & $\mathrm{~F}, 81$ & $\begin{array}{c}\text { ONAM } \\
\text { (estadio 3) } \\
\text { Alendronato } \\
\text { v.o. }\end{array}$ & Cuerpo & $\begin{array}{l}\text { Secuestrectomía } \\
+ \text { RAFR }\end{array}$ & $\begin{array}{l}\text { Exposición de } \\
\text { placa de } \\
\text { osteosíntesis }\end{array}$ & $\begin{array}{l}\text { Medidas de } \\
\text { higiene oral }\end{array}$ & $\begin{array}{c}\text { Cicatrización } \\
\text { completa }\end{array}$ & 18 meses \\
\hline 3 & $F, 65$ & ORN & Cuerpo & $\begin{array}{c}\text { Secuestrectomía } \\
+ \text { RAFR }\end{array}$ & $\begin{array}{l}\text { Exposición de } \\
\text { placa de } \\
\text { osteosíntesis }\end{array}$ & $\begin{array}{l}\text { Antibióticos y } \\
\text { medidas de } \\
\text { higiene oral. }\end{array}$ & $\begin{array}{l}\text { Muerte por } \\
\text { enfermedad }\end{array}$ & 6 meses \\
\hline 4 & $F, 68$ & ORN & Cuerpo & RAFR & - & $\begin{array}{c}\text { Cicatrización } \\
\text { completa }\end{array}$ & $\begin{array}{c}\text { Cicatrización } \\
\text { completa }\end{array}$ & 22 meses \\
\hline 5 & M, 65 & $\begin{array}{c}\text { CCE } \\
\text { (estadio IVa) }\end{array}$ & Cuerpo & $\begin{array}{c}\text { Resección + RAFR } \\
\text { + colgajo } \\
\text { microquirúrgico }\end{array}$ & $\begin{array}{l}\text { Necrosis de } \\
\text { colgajo }\end{array}$ & CMPM & $\begin{array}{l}\text { Muerte por } \\
\text { enfermedad }\end{array}$ & 6 meses \\
\hline 6 & F, 76 & $\begin{array}{c}\text { CCE } \\
\text { (estadio IVa) }\end{array}$ & Cuerpo & $\begin{array}{c}\text { Resección + RAFR } \\
\text { + CMPM }\end{array}$ & $\begin{array}{c}\text { Necrosis de } \\
\text { colgajo }\end{array}$ & CMPM & $\begin{array}{l}\text { Muerte por } \\
\text { enfermedad }\end{array}$ & 10 meses \\
\hline 7 & M, 85 & ORN & Cuerpo & $\begin{array}{l}\text { Antibióticos + } \\
\text { antisépticos } \\
\text { locales }\end{array}$ & $\begin{array}{c}\text { Fístula } \\
\text { orocutánea }\end{array}$ & - & $\begin{array}{c}\text { Cierre de } \\
\text { tejidos blandos }\end{array}$ & 11 meses \\
\hline 8 & $\mathrm{~F}, 70$ & $\begin{array}{c}\text { ONAM } \\
\text { (estadio 3) } \\
\text { Ácido } \\
\text { zoledrónico } \\
\text { i.v. }\end{array}$ & Cuerpo & Resección + RAFR & $\begin{array}{l}\text { Exposición de } \\
\text { placa }\end{array}$ & $\begin{array}{l}\text { Medidas de } \\
\text { higiene oral }\end{array}$ & $\begin{array}{l}\text { Persistencia de } \\
\text { dehiscencia } \\
\text { con tejido óseo } \\
\text { expuesto }\end{array}$ & 18 meses \\
\hline 9 & $\mathrm{~F}, 70$ & $\begin{array}{c}\text { ONAM } \\
\text { (estadio 3) } \\
\text { Alendronato } \\
\text { v.o. }\end{array}$ & Parasinfisiaria & RAFR & $\begin{array}{l}\text { Exposición de } \\
\text { placa }\end{array}$ & $\begin{array}{l}\text { Medidas de } \\
\text { higiene oral }\end{array}$ & $\begin{array}{c}\text { Cicatrización } \\
\text { completa }\end{array}$ & 19 meses \\
\hline 10 & M, 68 & $\begin{array}{c}\text { ONAM } \\
\text { (estadio 3) } \\
\text { Ácido } \\
\text { zoledrónico } \\
\text { i.v. }\end{array}$ & Ángulo & $\begin{array}{c}\text { Secuestrectomía } \\
+ \text { RAFR }\end{array}$ & - & - & $\begin{array}{l}\text { Cicatrización } \\
\text { completa }\end{array}$ & 12 meses \\
\hline 11 & $F, 84$ & $\begin{array}{c}\text { ONAM } \\
\text { (estadio 3) } \\
\text { Alendronato } \\
\text { v.o. }\end{array}$ & Cuerpo & RAFR & - & - & $\begin{array}{c}\text { Cicatrización } \\
\text { completa }\end{array}$ & 6 meses \\
\hline 12 & $\mathrm{~F}, 75$ & CCE & Cuerpo & $\begin{array}{c}\text { Resección + RAFR } \\
\text { + CMPM }\end{array}$ & $\begin{array}{c}\text { Fracaso de } \\
\text { osteosíntesis }\end{array}$ & $\begin{array}{l}\text { Recambio de } \\
\text { osteosíntesis }\end{array}$ & $\begin{array}{l}\text { Muerte por } \\
\text { enfermedad }\end{array}$ & 10 meses \\
\hline 13 & M, 82 & $\begin{array}{c}\text { ONAM } \\
\text { (estadio 3) } \\
\text { Ácido } \\
\text { Zoledrónico } \\
\text { i.v. }\end{array}$ & Parasinfisiaria & $\begin{array}{c}\text { Secuestrectomía } \\
+ \text { RAFR }\end{array}$ & $\begin{array}{l}\text { Dehiscencia y } \\
\text { exposición ósea }\end{array}$ & $\begin{array}{l}\text { Medidas de } \\
\text { higiene oral }\end{array}$ & $\begin{array}{l}\text { Cicatrización } \\
\text { completa }\end{array}$ & 14 meses \\
\hline 14 & $\mathrm{M}, 67$ & $\begin{array}{l}\text { Mieloma } \\
\text { múltiple }\end{array}$ & Cuerpo & RAFR & - & - & $\begin{array}{c}\text { Cicatrización } \\
\text { completa }\end{array}$ & 13 meses \\
\hline 15 & $F, 65$ & $\begin{array}{c}\text { Quiste } \\
\text { inflamatorio }\end{array}$ & Parasinfisiaria & $\begin{array}{c}\text { Quistectomía + } \\
\text { RAFR }\end{array}$ & - & - & $\begin{array}{c}\text { Cicatrización } \\
\text { completa }\end{array}$ & 17 meses \\
\hline
\end{tabular}

CCE: carcinoma de células escamosas. CMPM: colgajo del músculo pectoral mayor. i.v.: vía intravenosa. F: femenino. M: masculino. ONAM: osteonecrosis asociada a medicamentos. ORN: osteorradionecrosis. RAFR: reducción abierta y fijación rígida. v.o.: vía oral. 
Tabla II. Revisión de la bibliografía

\begin{tabular}{|c|c|c|c|c|}
\hline Autor & Año & $\mathrm{n}$ & Etiología & Tipo de estudio \\
\hline Millet y cols. ${ }^{9}$ & 1990 & 2 & ORN & Serie de casos \\
\hline MacMillan y cols. ${ }^{10}$ & 1991 & 1 & Granuloma eosinofílico & Presentación de caso \\
\hline Ezsiás y cols. ${ }^{1}$ & 1994 & 4 & $\begin{array}{l}\text { Quistes ( } 3 \text { casos) } \\
\text { CCE ( } 1 \text { caso) }\end{array}$ & Serie de casos \\
\hline Furutani y cols. ${ }^{11}$ & 1994 & 1 & Mieloma múltiple & Serie de casos \\
\hline Gerhards y cols. ${ }^{8}$ & 1998 & 30 & $\begin{array}{c}\text { ORN (9 casos) } \\
\text { Osteomielitis (6 casos) } \\
\text { Atrofia ( } 4 \text { casos) } \\
\text { Quistes ( } 3 \text { casos) } \\
\text { Tumores benignos ( } 3 \text { casos), MTTS (3 casos) } \\
\text { Mieloma múltiple (caso) } \\
\text { CCE (3 casos) }\end{array}$ & Serie de casos y revisión de bibliografía \\
\hline Kalantar y cols. ${ }^{12}$ & 1998 & 1 & QOA & Serie de casos \\
\hline O'Sullivan y cols. ${ }^{13}$ & 2006 & 1 & Osteomielitis & Presentación de caso \\
\hline Goddard y cols. ${ }^{14}$ & 2007 & 1 & QOA & Presentación de caso \\
\hline $\begin{array}{c}\text { Dos Anjos Pontual } \\
\text { y cols. }{ }^{15}\end{array}$ & 2007 & 1 & Granuloma eosinofílico & Presentación de caso \\
\hline Ogasawara y cols. ${ }^{16}$ & 2008 & 1 & Osteomielitis & Presentación de caso \\
\hline Coletti y Ord ${ }^{7}$ & 2008 & 44 & $\begin{array}{l}\text { ORN ( } 19 \text { casos) } \\
\text { Osteomielitis ( } 13 \text { casos) } \\
\text { ONAM ( } 4 \text { casos) } \\
\text { CCE ( } 4 \text { casos }) \\
\text { MTTS }(2 \text { casos }) \\
\text { Tumor benigno }(1 \text { caso) } \\
\text { Quiste }(1 \text { caso })\end{array}$ & Serie de casos \\
\hline Jham y cols. ${ }^{17}$ & 2008 & 1 & ORN & Presentación de caso \\
\hline $\begin{array}{c}\text { Wongchuensoontorn } \\
\text { y cols. }{ }^{18}\end{array}$ & 2009 & 3 & ONAM & Serie de casos \\
\hline Gallego y cols. ${ }^{19}$ & 2010 & 1 & ONAM & Presentación de caso \\
\hline Vigliaroli y cols. ${ }^{20}$ & 2010 & 1 & Osteomielitis & Presentación de caso \\
\hline Kuijpers y cols. ${ }^{21}$ & 2011 & 1 & ONAM & Presentación de caso \\
\hline Boffano y cols. ${ }^{2}$ & 2013 & - & - & Revisión de bibliografía \\
\hline Manor y cols. ${ }^{22}$ & 2013 & 1 & CIPA & Presentación de caso \\
\hline Imai y cols. ${ }^{23}$ & 2014 & 1 & ONAM & Presentación de caso \\
\hline Chell y cols. ${ }^{24}$ & 2015 & 1 & QOS & Presentación de caso \\
\hline Roldan y cols..$^{25}$ & 2015 & 1 & ONAM & Presentación de caso \\
\hline Oliveira y cols. ${ }^{26}$ & 2016 & 1 & Linfoma & Presentación de caso \\
\hline Jauhar y cols. ${ }^{27}$ & 2016 & 1 & Osteomielitis & Presentación de caso \\
\hline Carlsen y cols. ${ }^{4}$ & 2016 & 17 & $\begin{array}{c}\text { ORN (8 casos) } \\
\text { CCE }(3 \text { casos }), \\
\text { ONAM }(2 \text { casos }) \\
\text { Tumores benignos }(2 \text { casos }) \\
\text { Osteomielitis }(1 \text { caso }) \\
\text { Quiste }(1 \text { caso })\end{array}$ & Serie de casos \\
\hline
\end{tabular}

CCE: carcinoma de células escamosas. CIPA: insensibilidad congénita al dolor con anhidrosis. MTTS: metástasis. ONAM: osteonecrosis asociada a medicamentos. ORN: osteorradionecrosis. QOA: quiste óseo aneurismático. QOS: quiste óseo simple.

Estrategia de búsqueda: "mandibular fractures"[MeSH Terms] AND "pathological”[All Fields] AND ("humans"[MeSH Terms] AND English[lang]). 
En nuestro estudio el 67 \% de los casos ocurrió en mujeres, en contraposición a otras publicaciones, donde se informa una clara predilección por el sexo masculino $2,7,8$. Consideramos que los resultados obtenidos en nuestra serie no indican una predilección por el sexo femenino, sino que esta diferencia puede explicarse por la distribución sociodemográfica de nuestro país para esta franja etaria, en la que las mujeres representan un $66 \%$.

Las series de casos publicadas en la bibliografía acerca de fracturas patológicas de mandíbula son escasas y solo tres incluyen más de diez pacientes ${ }^{4,7,8}$.

\section{CONCLUSIÓN}

En nuestra serie encontramos que la etiología más frecuente fue la osteonecrosis asociada a medicamentos seguida por el CCE y la ORN. Estos pacientes pueden ser tratados de forma exitosa siguiendo los algoritmos propuestos para la población general, aunque la tasa de complicaciones es elevada (53\%). Los pacientes con fracturas patológicas por CCE fueron los que presentaron peor evolución y todos fallecieron en un periodo de seguimiento promedio de 8 meses. En coincidencia con lo observado por otros autores, las tasas de resolución completa de la fractura en pacientes con osteonecrosis asociada a medicamentos tratados mediante este algoritmo parecen ser satisfactorios.

\section{B I B L I O G R A F Í A}

1. Ezsiás A, Sugar AW. Pathological fractures of the mandible: a diagnostic and treatment dilemma. Br J Oral Maxillofac Surg. 1994;32(5):303-6. DOI: 10.1016/0266-4356(94)90051-5.

2. Boffano P, Roccia F, Gallesio C, Berrone S. Pathological mandibular fractures: a review of the literature of the last two decades. Dent Traumatol. 2013;29(3):185-96. DOI: 10.1111/edt.12028.

3. World Population Prospects - Population Division - United Nations. [citado 6 Jan 2018]. Disponible en: https://esa.un.org/unpd/wpp/ Graphs/Probabilistic/POP/65plus/

4. Carlsen A, Marcussen M. Spontaneous fractures of the mandible concept \& treatment strategy. Med Oral Patol Oral Cir Bucal. 2016;21(1):e88-94. DOI: 10.4317/medoral.20716.

5. Davis JW, Chung R, Juarez DT. Prevalence of comorbid conditions with aging among patients with diabetes and cardiovascular disease. Hawaii Med J. 2011;70(10):209-13.

6. Lin HS, Watts JN, Peel NM, Hubbard RE. Frailty and post-operative outcomes in older surgical patients: a systematic review. BMC Geriatr. 2016;16(1):157. DOI: 10.1186/s12877-016-0329-8.

7. Coletti $D$, Ord RA. Treatment rationale for pathological fractures of the mandible: a series of 44 fractures. Int J Oral Maxillofac Surg. 2008;37(3):215-22. DOI: 10.1016/j.ijom.2007.09.176.

8. Gerhards F, Kuffner HD, Wagner W. Pathological fractures of the mandible. A review of the etiology and treatment. Int J Oral Maxillofac Surg. 1998;27(3):186-90. DOI: 10.1016/s0901-5027(98)80007-6.

9. Millett DT, Chapple IL, Hirschmann PN, Corrigan AM. Septic osteoradionecrosis of the mandible associated with pathological fracture: report of two cases. Clin Radiol. 1990;41(6):408-10. DOI: 10.1016/s0009-9260(05)80602-6.

10. MacMillan AR, Oliver AJ, Radden BG, Lacy MF. Langerhans cell disease associated with pathological fracture of the mandible. Aust Dent J. 1991;36(6):451-5. DOI: 10.1111/j.1834-7819.1991. tb04725.x.

11. Furutani M, Ohnishi M, Tanaka Y. Mandibular involvement in patients with multiple myeloma. J Oral Maxillofac Surg. 1994;52(1):235. DOI: 10.1016/0278-2391(94)90007-8.

12. Kalantar Motamedi M. H. Aneurysmal bone cysts of the jaws: cli- nicopathological features, radiographic evaluation and treatment analysis of 17 cases. J Craniomaxillofac Surg. 1998;26(1):56-62. DOI: 10.1016/s1010-5182(98)80036-x.

13. O'Sullivan D, King P,Jagger D. Osteomyelitis and pathological mandibular fracture related to a late implant failure: A clinical report. J Prosthet Dent. 2006;95(2):106-10. DOI: 10.1016/j.prosdent.2005.10.014.

14. 14. Goddard R, Patel N. Aneurysmal bone cyst masquerading as unknown mandibular metastatic deposit causing pathological fracture. Dent Update. 2007;34(4):230-2, 234. DOI: 10.12968/ denu.2007.34.4.230.

15. Dos Anjos Pontual ML, da Silveira MM, de Assis Silva Lima F, Filho FW. Eosinophilic granuloma in the jaws. Oral Surg Oral Med Oral Pathol Oral Radiol Endod. 2007;104(6):e47-51. DOI: 10.1016/j.tripleo.2007.07.006.

16. Ogasawara T, Sano K, Hatsusegawa C, Miyauchi K, Nakamura M, Matsuura H. Pathological fracture of the mandible resulting from osteomyelitis successfully treated with only intermaxillary elastic guiding. Int J Oral Maxillofac Surg. 2008;37(6):581-3. DOI: 10.1016/j. ijom.2007.11.006.

17. Jham BC, Aguiar MC, Jham AC, Duraes GV, Fernandes AM. Successful conservative treatment of osteoradionecrosis of the mandible associated with pathological fracture. Minerva Stomatol. 2008;57(1-2):65-9.

18. Wongchuensoontorn C, Liebehenschel N, Wagner K, Fakler O, Gutwald R, Schmelzeisen $\mathrm{R}$, et al. Pathological fractures in patients caused by bisphosphonate-related osteonecrosis of the jaws: report of 3 cases. J Oral Maxillofac Surg. 2009;67(6):1311-6. DOI: 10.1016/j.joms.2008.12.030.

19. Gallego L, Junquera L, Pelaz A, Costilla S. Pathological mandibular fracture after simple molar extraction in a patient with osteogenesis imperfecta treated with alendronate. Med Oral Patol Oral Cir Bucal. 2010;15(6):e895-7. DOI: 10.4317/medoral.15.e895.

20. Vigliaroli E, Broglia S, Iacovazzi L, Maggiore C. Double pathological fracture of mandibula caused by actinomycotic osteomyelitis: a case report. Minerva Stomatol. 2010;59(9):507-17.

21. Kuijpers SC, van Roessel EW, van Merkesteyn JP. Unusual case of a conservatively treated pathological fracture after sequestrectomy in a patient with long-term oral bisphosphonate use. J Craniomaxillofac Surg. 2011;39(1):69-72. DOI: 10.1016/j.jcms.2010.03.022.

22. Manor E, Joshua BZ, Levy J, Brennan PA, Bodner L. Pathological fracture of the mandible in a paediatric patient with congenital insensitivity to pain with anhidrosis (CIPA). J Craniomaxillofac Surg. 2013;41(2):e39-41. DOI: 10.1016/j.jcms.2012.07.008.

23. Imai T, Michizawa M. "Phoenix jaw"-bone regeneration of the necrotic mandible between pathological fractures: an unusual but evocative course of bisphosphonate-related osteonecrosis. J Craniofac Surg. 2014;25(4):e333-6. DOI: 10.1097/SCS.0000000000000595.

24. Chell M, Idle M, Green J. Case report: an unusual finding of a solitary bone cyst in a patient with a fractured mandible. Dent Update. 2015;42(10):977-8. DOI: 10.12968/denu.2015.42.10.977.

25. Roldan CJ, Paniagua L. Complications of new medications. West $J$ Emerg Med. 2015;16(1):154-6. DOI: 10.5811/westjem.2014.10.23837.

26. Oliveira MR, Fernandes D, Gabrielli MA, Andrade CR, Navarro CM, Pereira-FilhoVA. Pathological mandibular fracture associated with diffuse large b-cell lymphoma in HIV-positive patient. J Craniofac Surg. 2016;27(8):2084-7. DOI: 10.1097/SCS.00000000000003058.

27. Jauhar P, Handley T, Hammersley N. A Pathological fracture of the mandible due to osteomyelitis following a full dental clearance. Dent Update. 2016;43(2):168-70, 173, 175. DOI: 10.12968/ denu.2016.43.2.168

28. Van den Bergh B, Heymans MW, Duvekot F, Forouzanfar T. Treatment and complications of mandibular fractures: a 10-year analysis. J Craniomaxillofac Surg. 2012;40(4):e108-11. DOI: 10.1016/j. jcms.2011.05.015.

29. Chen CL, Zenga J, Patel R, Branham G. Complications and Reoperations in Mandibular Angle Fractures. JAMA Facial Plast Surg. 2018;20(3):238-243. DOI: 10.1001/jamafacial.2017.2227.

30. Morrow BT, Samson TD, Schubert W, Mackay DR. Evidence-based medicine: Mandible fractures. Plast Reconstr Surg. 2014;134(6):138190. DOI: 10.1097/PRS.0000000000000717. 